\title{
Mixed finite element method of a seawater intrusion problem in confined aquifers
}

\author{
Ibtissam Medarhri ${ }^{1,2}$, Mohamed Farhloul ${ }^{3}$, Khalid Najib ${ }^{1,2}$, Soumia Slimani ${ }^{1, *}$ and Abdelmalek Zine ${ }^{4}$ \\ ${ }^{1}$ LERMA, Ecole Mohammadia d'Ingénieurs, université Mohammed V, Agdal-Rabat, Maroc. \\ ${ }^{2}$ LMAID, Ecole Nationale Supérieure des Mines de Rabat, 1000 Agdal,Maroc. \\ 3 Département de Mathématiques et de Statistique, Université de Moncton, Moncton, NB, E1A 3E9, Canada. \\ ${ }^{4}$ Institut Camille Jordan, Ecole centrale Lyon, 69134 Ecully, France.
}

\begin{abstract}
We consider a model mixing sharp and diffuse interface approach for the seawater intrusion phenomenon in confined aquifers. The aim of this work is to introduce and analyze a new mixed formulation, obtained by writing the problem into a matrix form, and introducing a new variable $\sigma=R(u) \nabla u$ representing the flux tensor of the primal variable $u=\left(h, \Phi_{f}\right)^{T}$. Here, h represents the depth of the salt/freshwater interface, $\Phi_{f}$, the hydraulic head of freshwater, and $\mathrm{R}(\mathrm{u})$ a symmetric and positive definite diffusion matrix. We show that the continuous problem is well-posed. For the time discretization of this new mixed formulation, we use a semi-implicit scheme, and we show that the problem is well posed.
\end{abstract}

${ }^{*}$ Corresponding author: soumaiaslim@gmail.com 


\section{Introduction}

In many countries and regions all over the globe, groundwater is considered the primary source of freshwater supply. Unfortunately, in coastal areas, a hydraulic exchange between groundwater and seawater may occur.

This exchange may arise for two main reasons: natural conditions such as the decline of the water table after a dry period or human impact such as intensive pumping. These factors lead to a decrease in the pressure of the water table, which, therefore, causes saltwater intrusion into coastal aquifers. Consequently, industrial and agricultural production may sustain significant damage. Thus, building a model, which simulates the movement of saltwater fronts in the coastal aquifer, is important for reasonable groundwater development and freshwater preservation. Within this context, several models based on numerical methods have been proposed and evaluated in the literature, see [1-3-6].

Considering the case of confined aquifers, we adopt a sharp/diffuse interface approach (see Fig. 1.). The domain is thus occupied by two immiscible fluids (freshwater and saltwater) separated by a sharp interface. For modeling the boundary conditions on the sharp interface, we use the Allen-Cahn model in the fluid/fluid context. We refer to [3], for more details on this approach .

The mathematical model associated with confined aquifers is given by a strongly coupled set of quasi-linear elliptic-parabolic equations. The considered unknowns are $h$ the depth freshwater/saltwater interface and $\Phi_{f}$, the hydraulic head. It must be noted that the global in time existence result is demonstrated in [4].

The use of the mixed sharp/diffuse interface approach provides a result of solution regularity. Indeed, the gradient of the solution is contained in the space
$L^{r}\left(0, T, W^{1, r}(\Omega)\right), r>2$. This result gives the uniqueness of the solution; we refer to [5] for more details. We propose, in this paper, a new mixed formulation of the problem. A time discretization of this new mixed formulation is based on a semi-implicit scheme. We show that the associated problem is well posed.

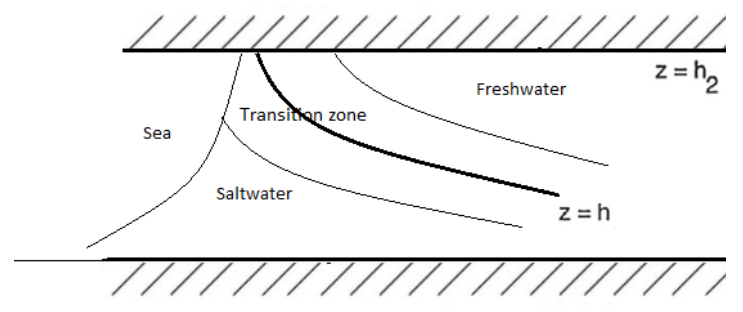

Fig.1 Description of the confined aquifer with a sharp/diffuse interface.

\section{Modelling}

We give, in this section, the model associated with a sharp/diffuse interface approach in a confined aquifer.

\subsection{Assumptions and notations}

The assumptions and results drawn in [4-5] are adopted.

We consider a bounded open domain of $R^{2}$ describing the projection of the porous medium on the horizontal plane with a boundary of class $C^{1}$. The time interval is $[0, T], T>0$ and we fix $\Omega_{T}=\Omega \times[0, T]$.

Moreover, we assume that the maximum principle is always verified and the hydraulic conductivity $K$ is a 
Homothety matrix. Therefore, the problem is formulated as follows:

$$
\begin{array}{r}
\phi \partial_{t} h+\nabla \cdot\left(K\left(h_{2}-h\right) \nabla \Phi_{f}\right)-\nabla \cdot\left(\alpha K\left(h_{2}-h\right) \nabla h\right) \\
-\phi \nabla \cdot(\delta \nabla h)=-Q_{s} \quad \text { in } \quad \Omega_{T}, \quad \text { (1) } \\
-\nabla \cdot\left(K h_{2} \nabla \Phi_{f}\right)+\nabla \cdot\left(\alpha K\left(h_{2}-h\right) \nabla h\right) \\
=Q_{f}+Q_{s} \quad \text { in } \Omega_{T}, \quad \text { (2) } \\
h=h_{D^{\prime}} \quad \Phi_{f}=\Phi_{f, D} \text { on } \Gamma \times[0, T] \\
h(x, 0)=h_{0}(x)
\end{array}
$$

where $\alpha$ is the parameter of density contrast, $\phi$ the porosity of the medium and $Q_{i}, i=f, s$ represents the external source terms corresponding to the pumping or recharge of fresh or saltwater into the aquifer, respectively.

The matrix form of the system (1-4) is given by:

$$
\left\{\begin{aligned}
M \partial_{t} u-\operatorname{div}(R(u) \nabla u) & =F & & \text { in } \Omega_{T} \\
u & =u_{D} & \text { on } & \Gamma \times[0, T], \\
u(0, .) & =u_{0} & & \text { in } \Omega,
\end{aligned}\right.
$$

where $u=\left(h, \Phi_{f}\right)^{T}$ and $R(u)$ is a symmetric positive definite matrix.

It should be noted that the analysis of problem (5) in the stationary case has already been done [7]. Thus, the results will be helpful to prove those in the non-stationary case.

\subsection{Mixed formulation}

We consider homogeneous Dirichlet conditions, $u_{D}=0$. without loss of generality. To derive a mixed formulation of the problem (5), we define the following spaces:

$$
X=[H(\operatorname{div}, \Omega)]^{2}, \quad Y=\left[L^{2}(\Omega)\right]^{2}
$$

and

$$
W(0, T)=\left\{v \in L^{2}\left(0, T, H_{0}^{1}(\Omega)\right) ; \partial_{t} v \in L^{2}\left(0, T, H^{-1}(\Omega)\right)\right\},
$$

where

$$
H(\operatorname{div}, \Omega):=\left\{w \in\left[L^{2}(\Omega)\right]^{2} ; \operatorname{div} w \in L^{2}(\Omega)\right\} .
$$

The spaces $X$ and $Y$ are equipped with the following norms:

$$
\begin{gathered}
\|\tau\|_{X}:=\left(\|\tau\|_{0, \Omega}^{2}+\|\operatorname{div} \tau\|^{2}\right)^{1 / 2}, \\
\|v\|_{Y}:=\|v\|_{0, \Omega^{\prime}}
\end{gathered}
$$

where $\|v\|_{0, \Omega}$ denotes the norm in $\left[L^{2}(\Omega)\right]^{N}, N$ is a positive integer.

When $N=2 \times 2$, this norm is associated with the inner product on $\left[L^{2}(\Omega)\right]^{2 \times 2}$

$$
(\sigma, \tau)=\int_{\Omega} \sigma: \tau, \text { where } \sigma=\left(\sigma_{i, j}\right), \tau=\left(\tau_{i, j}\right) \text { and } \sigma: \tau=\sum_{i, j} \sigma_{i, j} \tau_{i, j}
$$

Introducing the new variable $\sigma=R(u) \nabla u$, we may rewrite the problem (5) as follows:

$$
\begin{aligned}
& \left\{\begin{array}{rlrl}
M \partial_{t} u-\operatorname{div} \sigma & =F & & \text { in } \Omega_{T}, \\
\sigma-R(u) \nabla u & =0 & & \text { in } \Omega_{T}, \\
u & =u_{D} \text { on } & \Gamma \times[0, T] . \\
u(0, .) & =u_{0} & & \text { in } \Omega .
\end{array}\right. \\
& \sigma:=\sigma(t, x) \text { similarly, for } u \text { and } F \text {. }
\end{aligned}
$$

Above, and throughout the paper, (.,.) means the scalar product on $\left[L^{2}(\Omega)\right]^{N}, N$ is a positive integer.

\subsection{Some auxiliary results}

In this paragraph, we give beneficial results.

- $\quad R(u)$ is a symmetric and definite-positive matrix. We note $S(u)$ its inverse matrix.

- The matrix $S(u)$ is a symmetric, positive-definite matrix and L-Lipschitz,

$$
\begin{array}{r}
\|S(u)-S(v)\|_{L \infty} \leq L\|u-v\|, \\
\forall u, v \in Y .
\end{array}
$$

$$
(S(v) \tau, \tau) \geq c_{1}\|\tau\|_{0, \Omega^{\prime}}^{2} \forall v \in Y, \tau \in\left[L^{2}(\Omega)\right]^{2 \times 2},
$$


$(S(v) \sigma, \tau) \leq c_{2}\|\sigma\|_{0, \Omega}\|\tau\|_{0, \Omega^{\prime}} \forall v \in Y, \sigma, \tau \in\left[L^{2}(\Omega)\right]^{2 \times 2}$.

- $\quad$ There exists a positive constant $\beta_{1}>0$ such that,

$$
\begin{aligned}
& \operatorname{Sup}_{\tau \in X, \tau \neq 0} \frac{(d i v \tau, v)}{\|\tau\|_{0, \Omega}} \geq \beta_{1}\|v\|_{0,4, \Omega}, \\
& \quad \forall v \in\left[L^{4}(\Omega)\right]^{2} . \text { (10) }
\end{aligned}
$$

\section{Resolution of the continuous problem}

We have

$u \in W(0, T) \times L^{2}\left(0, T, L^{2}(\Omega)\right)$ and $F \in L^{2}(0, T, Y)$.

Then, using Green's formula, we look for

$u(t, x) \in E(0, T):=H^{1}\left(0, T, L^{2}(\Omega)\right) \times L^{2}\left(0, T, L^{2}(\Omega)\right)$

and

$$
\sigma(t, x) \in L^{2}(0, T, X)
$$

such that,

$$
\begin{cases}\left(M \partial_{t} u, v\right)-(\operatorname{div} \sigma, v) & =(F, v), \quad \forall v \in Y, \\ (S(u) \sigma, \tau)+(u, \operatorname{div} \tau) & =0, \quad \forall \tau \in X, \\ u(0, .) & =u_{0} \quad \text { in } \Omega,\end{cases}
$$

where $u_{0} \in\left[H_{0}{ }^{1}(\Omega)\right]^{2}$.

It's obvious to prove that problem (11) admits a solution $(u, \sigma)$, where $\sigma=R(u) \nabla u$ and $u$ is a solution of (6). For the uniqueness, we give the following result:

Theorem 1 Under the following assumptions:

- the solution of the initial problem is more regular, in the sense that $\nabla u(t) \in\left[L^{4}(\Omega)\right]^{2 \times 2}$,

- the initial condition $u_{0}$ and the second member $F$ are sufficiently small.
The problem (11) admits a unique solution $(u, \sigma)$.

Proof:

We assume that problem (11) admits two solutions $\left(u_{1}, \sigma_{1}\right),\left(u_{2}, \sigma_{2}\right)$. We put $\underline{u}=u_{1}-u_{2}$ and $\underline{\sigma}=\sigma_{1}-\sigma_{2}$. Then we have

$$
\begin{cases}\left(M \partial_{t} u_{1}, v\right)-\left(\operatorname{div} \sigma_{1}, v\right) & =(F, v) \quad \forall v \in Y, \\ \left(S\left(u_{1}\right) \sigma_{1}, \tau\right)+\left(u_{1}, \operatorname{div} \tau\right) & =0 \quad \forall \tau \in X, \\ u_{1}(0, .) & =u_{0} \quad \text { in } \Omega,\end{cases}
$$

and

$$
\begin{cases}\left(M \partial_{t} u_{2}, v\right)-\left(\operatorname{div}_{2}, v\right) & =(F, v) \quad \forall v \in Y, \\ \left(S\left(u_{2}\right) \sigma_{2}, \tau\right)+\left(u_{2}, \operatorname{div} \tau\right) & =0 \quad \forall \tau \in X \\ u_{2}(0, .) & =u_{0} \quad \text { in } \Omega .\end{cases}
$$

This implies

$$
\begin{gathered}
\left(M \partial_{t} \underline{u}, v\right)+(\operatorname{div} \underline{\sigma}, v)=0 \quad \forall v \in Y, \quad(12) \\
\left(S\left(u_{1}\right) \sigma_{1}-S\left(u_{2}\right) \sigma_{2}, \tau\right)+(\underline{u}, \operatorname{div} \tau)=0 \quad \forall \tau \in X .
\end{gathered}
$$

From (13), (7) and (9), we get

$$
\begin{aligned}
& (\underline{u}, \operatorname{div} \tau)=\left(S\left(u_{2}\right) \sigma_{2}-S\left(u_{1}\right) \sigma_{1}, \tau\right) \\
& =\left(\left(S\left(u_{2}\right)-S\left(u_{1}\right)\right) \sigma_{2}, \tau\right)-\left(S\left(u_{1}\right) \underline{\sigma}, \tau\right) \\
& \quad \leq\left(L C\|\hat{F}\|\|\underline{u}\|_{0,4, \Omega}+c_{2}\|\underline{\sigma}\|_{0, \Omega}\right)\|\tau\|_{0, \Omega},
\end{aligned}
$$

where $\|\hat{F}\|=\|F\|_{L^{2}(0, T, Y)}+\left\|u_{0}\right\|_{1,4, \Omega}$.

Using the Inf-Sup condition (10), we obtain

$$
\|\underline{u}\|_{0,4, \Omega} \leq \frac{c}{\beta_{1}-L C\|\hat{F}\|}\|\underline{\sigma}\|_{0, \Omega} .
$$

By fixing $t \in[0, T]$, we take $v=\underline{u}$ and $\tau=\underline{\sigma}$ in (12) and (13). We have

$$
\left(M \partial_{t} \underline{u}, \underline{u}\right)+c_{1}\|\underline{\sigma}\|_{0, \Omega}^{2} \leq L C \quad\|\hat{F}\|\|\underline{u}\|_{0,4, \Omega^{\prime}}
$$
then using (14) we get

$$
\left(M \partial_{t} \underline{u}, \underline{u}\right) \leq\left(\frac{c_{2} L C\|\hat{F}\|}{\beta_{1}-L C\|\hat{F}\|}-c_{1}\right)\|\underline{\sigma}\|_{0, \Omega}^{2}
$$


Moreover, we have

$$
\left(M \partial_{t} \underline{u}, \underline{u}\right)=\frac{\phi}{2} \frac{d}{d t}\|\underline{h}(t)\|_{0, \Omega}^{2},
$$

where $\underline{u}=\left(\underline{h}, \underline{\varphi}_{f}\right)$.

Let's set

then if

$$
g(t)=\frac{\phi}{2}\|\underline{h}(t)\|_{0, \Omega^{\prime}}^{2}
$$

$$
\|\hat{F}\| \leq \frac{\beta_{1} c_{1}}{L C\left(c_{1}+c_{2}\right)}
$$

$g(t)$ is decreasing, therefore

$$
g(t) \leq g(0)=0
$$

Thus,

$$
\left(M \partial_{t \underline{u}} \underline{u}\right)=0
$$

and from (14) and (15), we get

$$
\begin{gathered}
\|\underline{\sigma}\|_{0, \Omega}=0 \Rightarrow \sigma_{1}=\sigma_{2}, \\
\|\underline{u}\|_{0,4, \Omega}=0 \Rightarrow u_{1}=u_{2} .
\end{gathered}
$$

\section{Resolution of the semi-discrete problem}

Let $\Delta t:=\frac{T}{N}>0$ denote the time step size and define

$t_{n}=n \Delta t,(n=0,1,2, \ldots ., N)$ and $u^{n}$ the approximation of uat time $t_{n}$.

The semi-implicit in-time mixed formulation is as follows:

Find $\left(u^{n+1}, \sigma^{n+1}\right) \in Y \times X$, such that $u^{0}=u_{0}$ and

$$
(\mathcal{P})_{n+1}\left\{\begin{array}{l}
\left(\frac{1}{\Delta t} M u^{n+1}, v\right)-\left(\operatorname{div} \sigma^{n+1}, v\right)=\left(G^{n}, v\right) \quad \forall v \in Y, \\
\left(S\left(u^{n}\right) \sigma^{n+1}, \tau\right)+\left(u^{n+1}, \operatorname{div} \tau\right)=0 \quad \forall \tau \in X,
\end{array}\right.
$$

where $G^{n}=F\left(t^{n+1},.\right)+\frac{1}{\Delta t} u^{n}$
Theorem 2 A solution of $(P)_{n+1}$ exists and is unique.

\section{Proof:}

Let us consider the problem: Find $u^{n+1} \in\left[H_{0}{ }^{1}(\Omega)\right]^{2}$ such that,

$$
\begin{cases}\frac{1}{\Delta t} M u^{n+1}-\operatorname{div}\left(R\left(u^{n}\right) \nabla u^{n+1}\right) & =G^{n} \text { in } \Omega, \\ u^{n+1} & =0 \text { on } \Gamma .\end{cases}
$$

Using Green Formula, we get

$$
\begin{array}{r}
\frac{1}{\Delta t}\left(M u^{n+1}, v\right)+\left(R\left(u^{n}\right) \nabla u^{n+1}, \nabla v\right)=\left(G^{n}, v\right), \\
\forall v \in\left[H_{0}{ }^{1}(\Omega)\right]^{2}
\end{array}
$$

Using Lax-Milgram, we get that problem (18) has a unique solution. Then, by introducing $\sigma^{n+1}=R\left(u^{n}\right) \nabla u^{n+1}$, we get the problem $(P)_{n+1}$. Hence the existence of a solution $\left(u^{n+1}, \sigma^{n+1}\right)$.

For uniqueness, we assume that $(P)_{n+1}$ admits two solutions, $\left(u_{1}^{n+1}, \sigma_{1}^{n+1}\right),\left(u_{2}^{n+1}, \sigma_{2}^{n+1}\right)$ and by recursion on $n$ we prove that

$$
\left(u_{1}^{n+1}, \sigma_{1}^{n+1}\right)=\left(u_{2}^{n+1}, \sigma_{2}^{n+1}\right)
$$

- For $n=0$, let's prove that

$$
\left(u_{1}^{1}, \sigma_{1}^{1}\right)=\left(u_{2}^{1}, \sigma_{2}^{1}\right)
$$

We have, for $i=1,2$ and $G^{0}=F\left(t^{1},.\right)+u^{0}$,

$$
\left\{\begin{array}{l}
\left(\frac{1}{\Delta_{t}} M u_{i}^{1}, v\right)-\left(\operatorname{div} \sigma_{i}^{1}, v\right)=\left(G^{0}, v\right) \quad \forall v \in Y, \\
\left(S\left(u^{0}\right) \sigma_{i}^{1}, \tau\right)+\left(u_{i}^{1}, \operatorname{div} \tau\right)=0 \quad \forall \tau \in X
\end{array}\right.
$$

Let $\underline{u}^{1}=u_{1}{ }^{1}-u_{2}{ }^{1}$ and $\underline{\sigma}^{1}=\sigma_{1}{ }^{1}-\sigma_{2}{ }^{1}$, we have

$$
\left\{\begin{array}{lll}
\left(\frac{1}{\Delta_{t}} M \bar{u}^{1}, v\right)-\left(\operatorname{div} \bar{\sigma}^{1}, v\right) & =0 & \forall v \in Y \\
\left(S\left(u^{0}\right) \bar{\sigma}^{1}, \tau\right)+\left(\bar{u}^{1}, \operatorname{div} \tau\right) & =0 & \forall \tau \in X
\end{array}\right.
$$


we take $v=\underline{u}^{1}$ and $\tau=\underline{\sigma}^{1}$ in (19), we get

$$
\underbrace{\left(\frac{1}{\Delta_{t}} M \bar{u}^{1}, \bar{u}^{1}\right)}_{\geq 0}+\left(S\left(u^{0}\right) \bar{\sigma}^{1}, \bar{\sigma}^{1}\right)=0
$$

This implies that,

$$
c_{1}\left\|\underline{\sigma}^{1}\right\|_{0, \Omega}^{2} \leq\left(S\left(u_{0}\right) \underline{\sigma}^{1}, \underline{\sigma}^{1}\right)=0
$$

thus,

$$
\left\|\underline{\sigma}^{1}\right\|_{0, \Omega}=0 \Rightarrow \sigma_{1}{ }^{1}=\sigma_{2}{ }^{1} .
$$

As well,

$$
\left(\underline{u}^{1}, \operatorname{div} \tau\right)=0,
$$

and by using the Inf-Sup condition (10), we get

$$
\left\|\underline{u}^{1}\right\|_{0,4, \Omega}=0 \Rightarrow u_{1}^{1}=u_{2}^{1}
$$

- Assuming that

$$
\left(u_{1}^{n}, \sigma_{1}^{n}\right)=\left(u_{2}^{n}, \sigma_{2}^{n}\right) \text {, }
$$

we obtain, with the same way as

$$
\begin{aligned}
& \left(u_{1}{ }^{1}, \sigma_{1}{ }^{1}\right)=\left(u_{2}{ }^{1}, \sigma_{2}{ }^{1}\right) \text {, that } \\
& \left(u_{1}^{n+1}, \sigma_{1}{ }^{n+1}\right)=\left(u_{2}^{n+1}, \sigma_{2}{ }^{n+1}\right) .
\end{aligned}
$$

\section{Results and conclusion}

Using a sharp/diffuse interface approach, we propose a new formulation of the seawater intrusion problem in confined aquifers. The mathematical analysis of the problem is based on the regularity of the solution, $\nabla u(t) \in\left[L^{4}(\Omega)\right]^{2 \times 2}$, and the Inf-Sup condition in $\left[L^{4}(\Omega)\right]^{2}$.

A time discretization of this new mixed formulation is based on a semi-implicit scheme. We prove that the associated problem is well posed.

In a future work, we will show the convergence result of the semi-implicit scheme as well as an error estimation result for the case of the fully discretized problem.

\section{REFERENCES}

[1] Bear, J.: Dynamics of Fluids in Porous Media. American Elsevier (1972).

[2] Brezzi, F., Fortin, M.: Mixed and Hybrid Finite Element Methods. Springer (1991).

[3] Choquet, C., Diedhiou, M., Rosier, C.: Derivation of a sharp-diffuse interfaces model for seawater intrusion in a free aquifer. Numerical simulations. Mathematics and Computers in Simulation 76, 138-158 (2016).

[4] Choquet, C., Li, J., Rosier, C.: Global existence for seawater intrusion models: Comparison Between sharp interface and sharp-diffuse interface approaches. Electronic Journal of differential Equations pp. 1-27 (2015)

[5] Choquet, C., Li, J., Rosier, C.: Uniqueness for cross-diffusion systems issuing from seawater intrusion problems. Electronic Journal of Differential Equations pp. 1-22 (2017)

[6] Najib, K., Rosier, C.: On the global existence for a degenerate elliptic-parabolic seawater intrusion problem. Mathematics and Computers in Simulation 81, 2282-2295 (2011).

[7] Slimani, S. Farhloul, M. Medarhri, I. Najib, K. Zine, A.: Mixed formulation of a stationary seawater intrusion problem in confined aquifers. (2021). Working paper or preprint, 